\title{
REGULAR MECHANICAL TRANSFORMATION OF ROTATIONS INTO TRANSLATIONS: PART 1. KINEMATIC ANALYSIS AND DEFINITION OF THE BASIC CHARACTERISTICS
}

\author{
Emilia Abadjieva $^{1 *}$, VAlentin Abadjiev ${ }^{2}$ \\ ${ }^{1}$ Graduate School of Engineering and Resource Science, Faculty of \\ Engineering and Resource Science, Akita University, Tegatagakuen-machi \\ 1-1, Akita, Japan \\ ${ }^{2}$ Institute of Mechanics, Bulgarian Academy of Sciences, Acad. G. Bonchev \\ Str., bl. 4, 1113 Sofia, Bulgaria
}

[Received 13 March 2017. Accepted 26 June 2017]

\begin{abstract}
The science that study the processes of motions transformation upon a preliminary defined law between non-coplanar axes (in general case) axes of rotations or axis of rotation and direction of rectilinear translation by three-link mechanisms, equipped with high kinematic joints, can be treated as an independent branch of Applied Mechanics. It deals with mechanical behaviour of these multibody systems in relation to the kinematic and geometric characteristics of the elements of the high kinematic joints, which form them. The object of study here is the process of regular transformation of rotation into translation. The developed mathematical model is subjected to the defined task for studying the sliding velocity vector function at the contact point from the surfaces elements of arbitrary high kinematic joints. The main kinematic characteristics of the studied type motions transformation (kinematic cylinders on level, kinematic relative helices (helical conoids) and kinematic pitch configurations) are defined on the bases of the realized analysis. These features expand the theoretical knowledge, which is the objective of the gearing theory. They also complement the system of kinematic and geometric primitives, that form the mathematical model for synthesis of spatial rack mechanisms.
\end{abstract}

KEY WORDS: Motion transformation, sliding velocity, vector function, scalar and vector field, kinematic cylinder of level, kinematic helical conoid, kinematic pitch configurations.

\footnotetext{
${ }^{*}$ Corresponding author e-mail: abadjieva@gipc.akita-u.ac.jp
} 


\section{INTRODUCTION}

The scale of the development of the world industrial manufacture and the application of different types mechanical motions converters in it, determine the necessity of: (1) build an exact strategy for choosing the right type device from a functional view point; (2) their rapid and effective implementation into practice.

The choice of the optimal type gear transmission is connected with determining the common section of the defined two basic groups of requirements. This is realized by comparison of the geometric, kinematic and dynamic characteristics, which together determine their exploitation properties and with the technological characteristics, in terms of their manufacturing.

Togeather with this first task, which solution is primarily based on the known types of mechanical gears (having an established industrial application), it is necessary the development of the science of gear mechanisms to create preconditions for studying new or little-known characteristics of mechanical multibody systems, that are oriented towards motions transformation in accordance with preliminary given laws. This is a premise for creating new gear transmissions, which would satisfy various requirements of the industrial practice more complete and more accurate. The solution of the second task is searched among the creating of the mathematical models that describe the physics of the different regular motions transformation, as well as in creating adequate approaches to the synthesis and design of the multibody systems. This involves approaches, which are based on analytical methods for studying different types of gear mechanisms, taking into account their geometric-kinematic characteristics. The results, contained in the mentioned above studies, are appropriate to be sufficiently general from a theoretical viewpoint. At the same time, they have to be structured in a manner, allowing consideration of the specific characteristics of the concrete type transmissions.

In this study, an attempt is made to define briefly some parts of the mentioned problems, by realizing a study of the processes of regular spatial motions transformation of type "rotation into translation", oriented to the kinematic analysis of their nature [1,2]. The creation and usage of adequate mathematical models are intended for analytical defining and studying the specific characteristics of the researched type motions transformation. These features are elements of the "theory of gearing" - a basic direction of "the science of gear mechanisms". Essentially, the theory of gearing is a theory of high kinematic joints, taking into account the specifics of their application in gear drives. The theory of gearing deals with the common principles, which control the motions transformation, by means of three-link mechanisms with high kinematic joints, in all possible placements of the specific type axes of rotations and directrixes of rectilinear translation motion. This gearing theory offers an apparatus for analytical and computer study of different types of gearings. 


\section{KINEMATIC THEORY OF SPATIAL TRANSFORMATION OF ROTATION INTO RECTILINEAR TRANSLATION}

2.1. STRUCTURE, GEOMETRIC AND KINEMATIC RELATION BETWEEN THE PROCESSES OF SPATIAL TRANSFORMATION OF "ROTATION INTO ROTATION $(R \leftrightarrow R)$ " AND "ROTATION INTO TRANSLATION $(R \leftrightarrow T)$ "

The wide spread in mechanics devices for motions transformation are three-link mechanisms, which movable links are set of discreet conjugate surfaces, forming high kinematic joints, when the motions transformation is realized [3-5]. Depending on the quality characteristics of the realized spatial motions transformation, they are divided into two types: gear mechanisms with crossed axes (hyperboloid gear set), realizing transformation of type $(R \leftrightarrow R)$ (rotation into rotation); spatial rack drives, realizing motions transformation of type $(R \leftrightarrow T)$ (rotation into translation and vice versa) [6].

Through this study, the structure-geometric similarity of the spatial gear mechanisms, which ensure the motions transformation $(R \leftrightarrow R)$ and $(R \leftrightarrow T)$, is illustrated. First, the basic kinematic parameters of the spatial transformations "rotation into rotation $(R \leftrightarrow R)$ " and "rotation into translation $(R \leftrightarrow T)$ " will be defined.

The transformation of type $(R \leftrightarrow R)$ is a such type, in which it is realized the transformation of motion with angular velocity $\bar{\omega}_{1}$ around a fixed in space axis $1-1$ into motion with angular velocity $\bar{\omega}_{2}$, around another fixed in space axis $2-2$ (which is crossed with the axis $1-1$ ). This is realized by means of high kinematic joints $\left(\Sigma_{1}: \Sigma_{2}\right)$.

The kinematic scheme of this type transformation, illustrated on Fig. 1a, is characterized with the following kinematic conditions:

$$
\begin{aligned}
& \omega_{1}=\text { constant }, \quad \omega_{2}=\text { constant } \\
& \frac{\omega_{1}}{\omega_{2}}=i_{12}=\frac{1}{\omega_{2} / \omega_{1}}=\frac{1}{i_{21}}=\text { constant }
\end{aligned}
$$

where $\omega_{1}$ is the magnitude of angular velocity vector $\bar{\omega}_{1} ; \omega_{2}-$ the magnitude of angular velocity vector $\bar{\omega}_{2} ; i_{12}, i_{21}$ - law of transformation $R \leftrightarrow R$ (velocity ratio).

The distance between the rotations axes $1-1$ and $2-2$ is $a_{w}=$ constant, and $\angle\left(\bar{\omega}_{1}, \bar{\omega}_{2}\right)=\delta=$ constant is the crossed angle of the axes of rotation.

The transformation of rotation motion around fixed in space axis $1-1$, realized with angular velocity $\bar{\omega}_{1}$ into translation motion with velocity $\bar{V}_{2}$ along the direction $2 t-2 t$, (placed in the space against axis $1-1$, under an angle $\angle\left(\bar{\omega}_{1}, \bar{V}_{2}\right)=\delta_{r}=$ constant), by means of surfaces $\Sigma_{1}$ and $\Sigma_{2}$, is called transformation of type ( $R \leftrightarrow$ $T)$. The kinematic scheme of this type transformation is shown in Fig. $1 \mathrm{~b}$ and it is 


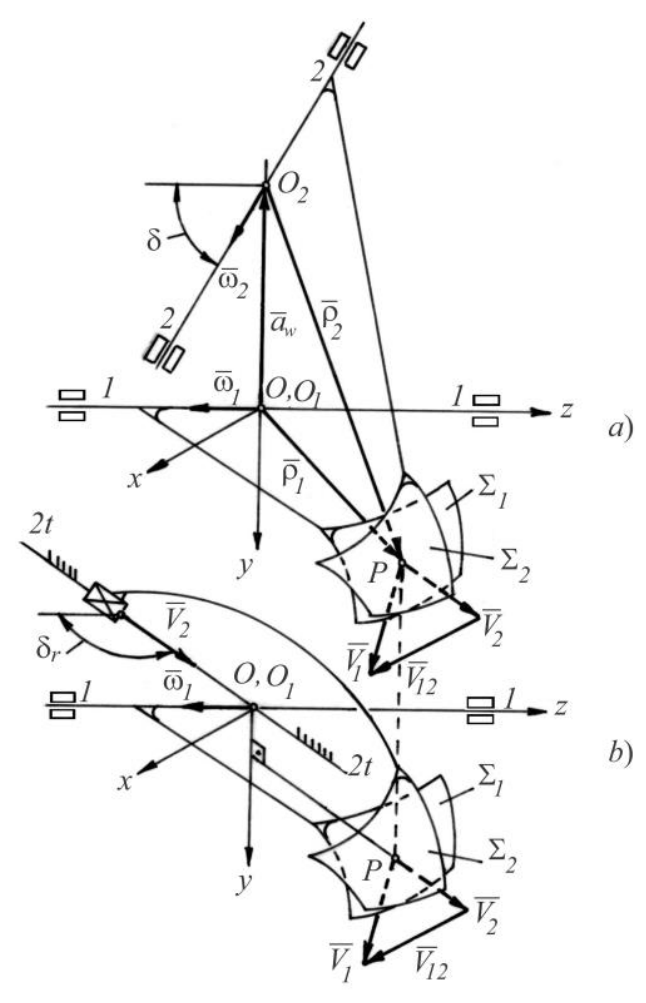

Fig. 1. Structural transition from hyperboloid gear transmission into spatial rack drive: a) kinematic scheme of gear mechanism, realizing transformation of type $(R \leftrightarrow R)$; b) kinematic scheme of gear mechanism, realizing transformation of type $(R \leftrightarrow T)$.

characterized with the following kinematic conditions [1,2]:

$$
\begin{aligned}
& \omega_{1}=\text { constant }, \quad V_{2}=\text { constant }, \\
& \frac{\omega_{1}}{V_{2}}=j_{12}=\frac{1}{V_{2} / \omega_{1}}=\frac{1}{j_{21}}=\text { constant }
\end{aligned}
$$

where $V_{2}$ - value of the translation velocity vector $\bar{V}_{2} ; j_{12}, j_{21}-$ law of transformation ( $R \leftrightarrow T$ ). Here, it will be noted that $i_{12}, i_{21}$ are non-dimensional numbers, $j_{12}$ has a dimension $[\mathrm{rad} / \mathrm{mm}]$, and $j_{21}$ is with dimension [mm/rad], since $\omega_{i}(i=1,2)$ are with dimension $[\mathrm{rad} / \mathrm{s}]$, and $V_{1}$ is with dimension $[\mathrm{mm} / \mathrm{s}]$. The spatial transformation of type $(R \leftrightarrow T)$ can be considered as a special case of the spatial transformation $(R \leftrightarrow R)$. In other words, the spatial rack drive can be considered as a border case of hyperboloid gear, i.e. it is obtained from a three-link spatial gear mechanism with crossed axes, under the following structural and kinematic changes (according to 
Fig. $1 \mathrm{a}$ and $b)$ :

- Increasing to "infinity" the number of the teeth related to one of the rotating links; for example increasing to "infinity" the number of the geometric elements $\Sigma_{2}$ of the high kinematic joint $\left(\Sigma_{1}: \Sigma_{2}\right)$;

- the number of mutually meshed tooth surfaces $\Sigma_{1}$ and $\Sigma_{2}$, i.e. the number of the high kinematic joints $\left(\Sigma_{1}: \Sigma_{2}\right)$ remains finite;

- the axis of rotation $2-2$ of the surfaces $\Sigma_{2}$ is moved into the "infinity";

- the rotation motion of $\Sigma_{2}$ around $2-2$ with an angular velocity $\bar{\omega}_{2}$ is transformed into a translational one with velocity $\bar{V}_{2}$.

\subsection{VeCtOR ANALYSIS OF THE SLIDING VELOCITY VECTOR}

Let's determine the sliding velocity vector $\bar{V}_{12}$ in the contact point $P$ of the high kinematic joint $\left(\Sigma_{1}: \Sigma_{2}\right)$, that realizes transformation of type $(R \leftrightarrow T)$ ), illustrated in Fig. 2. The elements $\Sigma_{1}$ and $\Sigma_{2}$ of the high kinematic joint $\left(\Sigma_{1}: \Sigma_{2}\right)$, are parametrically defined in the fixed co-ordinate system $S$ :

$$
\begin{array}{ll}
\bar{\rho}_{1, s}=\bar{\rho}_{1, s}\left(u_{1}, \vartheta_{1}, \varphi_{1}\right), & \bar{\rho}_{2, s}=\bar{\rho}_{2, s}\left(u_{2}, \vartheta_{2}, j_{21} \varphi_{1}\right), \\
\bar{n}_{1, s}=\bar{n}_{1, s}\left(u_{1}, \vartheta_{1}, \varphi_{1}\right), & \bar{n}_{2, s}=\bar{n}_{2, s}\left(u_{2}, \vartheta_{2}, j_{21} \varphi_{1}\right),
\end{array}
$$

where $\bar{\rho}_{i, s}$ is a radius-vector of the contact point $P$, as a point from $\Sigma_{i} ; \bar{n}_{i, s}-$ normal vector to the $\Sigma_{i}$ at the same point; $u_{i}, \vartheta_{i}$-independent parameters, determining the position of point $P$ on $\Sigma_{i} ; j_{21} \varphi_{1}$ - translational displacement of the link 2, corresponding to the rotation of the link 1 at an angle $\varphi_{1}$.

For $\Sigma_{1}$ and $\Sigma_{2}$, the following conditions are fulfilled, since $\Sigma_{1}$ and $\Sigma_{2}$ are elements of high kinematic joints, which take part in the process of motions transformation $(R \leftrightarrow T)$ :

$$
\begin{aligned}
& \bar{\rho}_{1, s}\left(u_{1}, \vartheta_{1}, \varphi_{1}\right)=\bar{\rho}_{2, s}\left(u_{2}, \vartheta_{2}, j_{21} \varphi_{1}\right), \\
& \bar{n}_{1, s}\left(u_{1}, \vartheta_{1}, \varphi_{1}\right)=\bar{n}_{2, s}\left(u_{2}, \vartheta_{2}, j_{21} \varphi_{1}\right), \\
& \dot{\bar{\rho}}_{1, s}\left(u_{1}, \vartheta_{1}, \varphi_{1}\right)=\dot{\bar{\rho}}_{2, s}\left(u_{2}, \vartheta_{2}, j_{21} \varphi_{1}\right), \\
& \dot{\bar{n}}_{1, s}\left(u_{1}, \vartheta_{1}, \varphi_{1}\right)=\dot{\bar{n}}_{2, s}\left(u_{2}, \vartheta_{2}, j_{21} \varphi_{1}\right) .
\end{aligned}
$$

where $\dot{\bar{\rho}}_{i, s}$ is an absolute velocity vector of the contact point $P$, as a point from $\Sigma_{i}$; $\dot{\bar{n}}_{i, s}$ - an absolute velocity vector of the tip of normal vector $\bar{n}_{i, s}$ in the contact point $P$, as a point from $\Sigma_{i}$. 
In accordance with the given scheme of motions transformation of type $(R \leftrightarrow T)$ in Fig. 2, it is characterized with the following conditions [7]:

$$
\begin{aligned}
& \omega_{1}=\text { constant }, \quad V_{2}=\text { constant } \\
& \delta_{r}=\angle\left(\bar{\omega}_{1}, \bar{V}_{2}\right)=\pi-\Sigma_{r}=\text { constant } \\
& j_{12}=\frac{\omega_{1}}{V_{2}}=\frac{1}{j_{21}}=\text { constant }
\end{aligned}
$$

where $\omega_{1}$ is the value of the angular velocity vector $\bar{\omega}_{1}$ of the rotating link $i=1 ; V_{2}$ - is the value of the translation velocity vector $\bar{V}_{2}$ of link $i=2 ; \delta_{r}$ is crossed angle of the vectors, that characterize motions transformation; $\Sigma_{r}-$ an angle between the motions' axes (axis of rotations and direction of the rectilinear translation) of the transformation $(R \leftrightarrow T) ; j_{21}$ - velocity ratio for transformation of type $(R \leftrightarrow T)$.

As it was said in the beginning, the studied motions transformation is realized by means of high kinematic joint $\left(\Sigma_{1}: \Sigma_{2}\right)$, which elements $\Sigma_{1}$ and $\Sigma_{2}$, at a given moment have a contact point $P$ (the point of tangential contact $P$ can belong to the conjugate instantaneous contact line $D_{12}$ ). The second movable link $i=2$ obtains a translation velocity $\bar{V}_{2}$, when rotation with angular velocity $\bar{\omega}_{1}$ is applied to the link $i=1$. According to (6), the condition $V_{2}=j_{21} \omega_{1}$ is accomplished for the value of $\bar{V}_{2}$. Hence, the joint $\left(\Sigma_{1}: \Sigma_{2}\right)$, shown in Fig. 2 is kinematically conjugated.

The study is realized by the right-hand co-ordinate systems $S(O, x, y, z)$, $S_{0}\left(O_{0}, x_{0}, y_{0}, z_{0}\right)$ and $S_{i}\left(O_{i}, x_{i}, y_{i}, z_{i}\right),(i=1,2)$ (see Fig. 2). The first two coordinate systems $S$ and $S_{0}$ are fixed co-ordinate systems (connected with the posture of the mechanism) and the second ones $-S_{1}$ and $S_{2}$ are connected with the movable links of the mechanism.

The vector analysis of the vector function of the sliding velocity vector (relative velocity) in contact point $P$ of the conjugated surfaces $\Sigma_{1}$ and $\Sigma_{2}$ of the high kinematic joint (shown in Fig. 2) is based on the first equation of (5) [1], when an adequate mathematical model is constructed:

$$
\bar{V}_{12}=\bar{V}_{12}(x, y, z)=\bar{V}_{1}-\bar{V}_{2}=\bar{\omega}_{1} \times \bar{\rho}_{1}-\bar{V}_{2} .
$$

According to the condition for transformation (6), and the given in Fig. 2 symbols, for the above function written in co-ordinate system $S(O, x, y, z)$, it is obtained

$$
\begin{aligned}
& \bar{V}_{12}=\bar{V}_{12}(x, y, z)=V_{12, x} \bar{i}+V_{12, y} \bar{j}+V_{12, z} \bar{k}, \\
& V_{12, x}=-y, \quad V_{12, y}=x+j_{21} \sin \Sigma_{r}, \\
& V_{12, z}=j_{21} \cos \Sigma_{r},
\end{aligned}
$$

where $V_{12, x}, V_{12, y}, V_{12, z}$ are co-ordinates of $\bar{V}_{12}$ in the co-ordinate system $S$. 


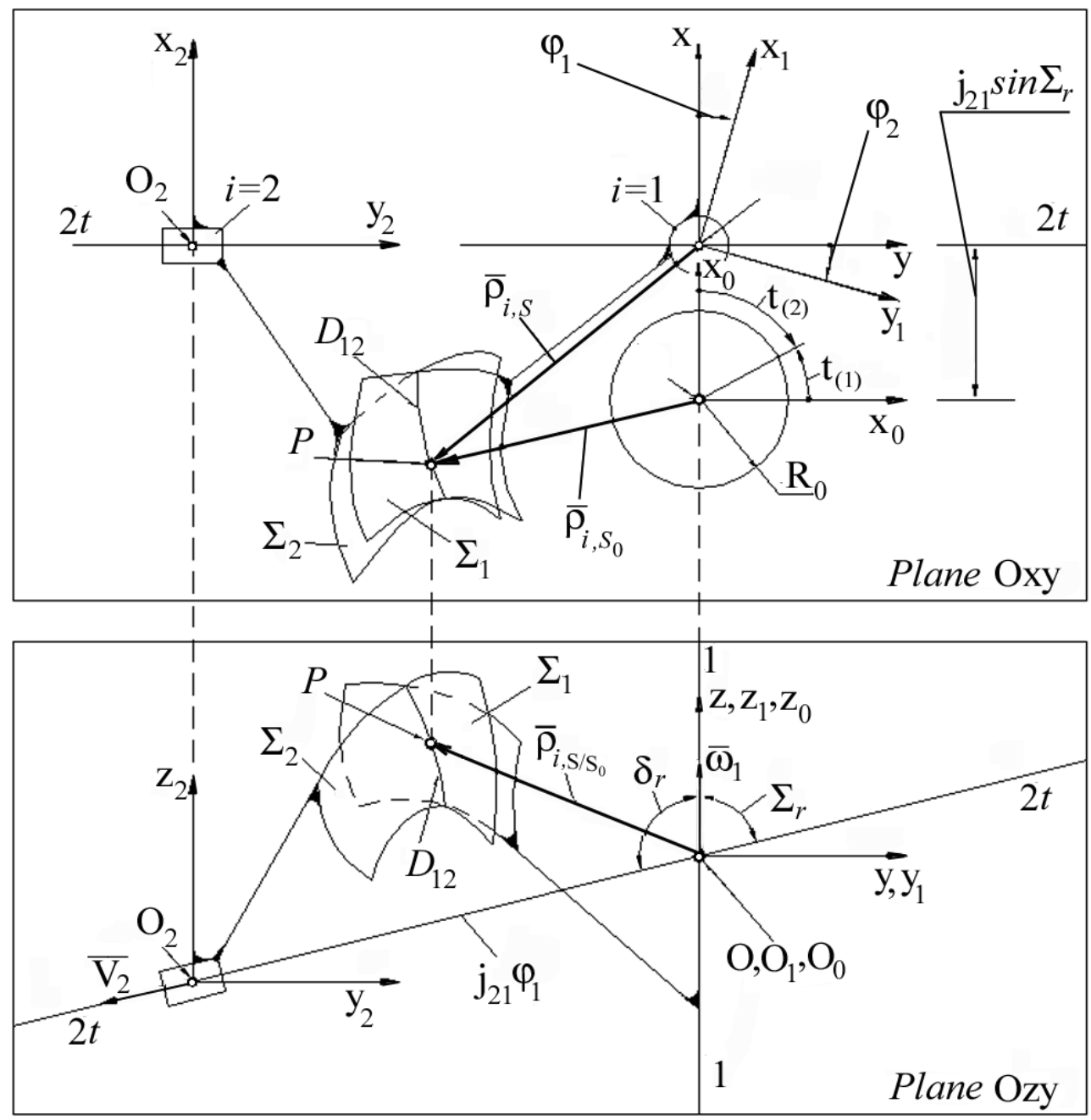

Fig. 2. Geometric-kinematic scheme of mechanical motions transformation of type $(R \leftrightarrow$ $T)$ and vice versa: $S(O, x, y, z)$ and $S_{0}\left(O_{0}, x_{0}, y_{0}, z_{0}\right)$ - fixed co-ordinate systems; $S_{i}\left(O_{i}, x_{i}, y_{i}, z_{i}\right)$ - co-ordinate systems, connected with movable links $i=1,2 ; \Sigma_{i}(i=1,2)$ - geometric elements of the high kinematic joints $\left(\Sigma_{1}: \Sigma_{2}\right) ; D_{12}-$ instantaneous contact line between $\Sigma_{1}$ and $\Sigma_{2} ; P$ - instantaneous contact point between $\Sigma_{1}$ and $\Sigma_{2} ; 1-1-$ axis of rotation; $2 t-2 t-$ direction of rectilinear translation; $\bar{\omega}_{1}-$ angular velocity vector of the rotating link $i=1 ; \bar{V}_{2}$ - translation velocity vector of link $i=2 ; \bar{\rho}_{i, s / s_{0}}$ - radius-vector of the contact point $P$, as a point from $\Sigma_{1}$ and $\Sigma_{2}$, written in the co-ordinate systems $S$ and $S_{0}$; $\delta_{r}$ - crossed angle between vectors, that characterize the motions transformation; $\Sigma_{r}-$ offset angle of the rack drive; $\varphi_{1}-$ meshing parameter. 
In equation (8), without disturbing the community of arguments, it is accepted $\omega_{1}=1 \mathrm{rad} / \mathrm{s}$ and therefore $V_{2}=j_{21} \mathrm{~mm} / \mathrm{s}$. The vector analysis of the vector function (8) is realized by defining its scalar and vector field.

Scalar field of the relative velocity $\bar{V}_{12}$ of the conjugate tooth surfaces $\Sigma_{1}$ and $\Sigma_{2}$ in their common contact point $P$ (considered as a point from the fixed space $S(O, x, y, z))$ is a scalar function of the type

$$
V_{12}=V_{12}(x, y, z)=\sqrt{V_{12, x}^{2}+V_{12, y}^{2}+V_{12, z}^{2}},
$$

with its definition area. Hence, the surfaces of type

$$
V_{12}(x, y, z)=V_{c}, \quad V_{c}=\text { constant }
$$

are surfaces of level of the studied scalar field and they define it geometrically [9].

It is easy to determine their canonical type in the static co-ordinate system $S_{0}\left(O_{0}, x_{0}, y_{0}, z_{0}\right)$ from (8), (9) and (10)

$$
\begin{aligned}
& x_{0}^{2}+y_{0}^{2}=R_{0}^{2}, \\
& z_{0}=H, \quad H \in(-\infty,+\infty),
\end{aligned}
$$

where $x_{0}=x+j_{21} \sin \Sigma_{r}, y_{0}=y, z_{0}=z, R_{0}=\sqrt{V_{c}^{2}-\left(j_{21} \cos \Sigma_{r}\right)^{2}}$.

Equations (11) show that the surfaces of level of the defined scalar field $V_{12}=$ $V_{12}\left(x_{0}, y_{0}, z_{0}\right)$ represent a family of coaxial cylinders with a family parameter $V_{c}$. These cylinders are called kinematic cylinders of level, by analogy with hyperboloid gears [8]. The geometric axis of the family coaxial cylinders is the axis $O_{0} z_{0}$ of the co-ordinate system $S_{0}\left(O_{0}, x_{0}, y_{0}, z_{0}\right)$, which in the fixed co-ordinate system $S(O, x, y, z)$ has the following equations:

$$
x=-j_{21} \sin \Sigma_{r}, \quad y=0, \quad z=H, \quad H \in(-\infty,+\infty) .
$$

In accordance with equation (11), the surfaces of level of the studied scalar field will exist, if the parameter $V_{c}$ is chosen so that it fulfills the condition

$$
V_{c} \geq j_{21} \cos \Sigma_{r}
$$

The parameter $V_{c}$ multiplied by $\omega_{1}^{-1}$, defines the module of the sliding velocity vector $\bar{V}_{12}$. Every point that belongs to a concrete kinematic cylinder of level is characterized with this parameter $V_{c}$. The given velocity ratio $j_{21}$ is realized by every point of the cylinders of level. When the equality in (13) is fulfilled, then the equation (11) is transformed into system of equations (12), which describe the zero kinematic cylinder of level. It contains those points from the fixed space, which are 


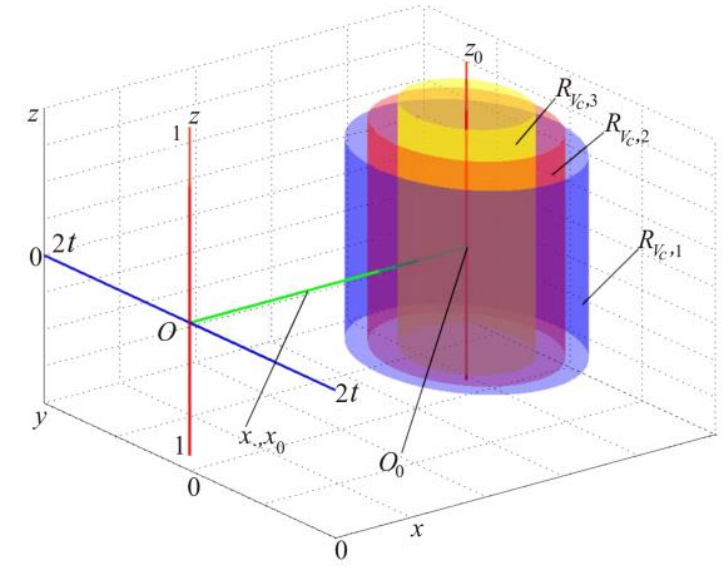

Fig. 3. Set of kinematic cylinders: $R_{V_{c}, 1}$ corresponds to the $V_{c, 1}=23 \mathrm{~mm} / \mathrm{rad} ; R_{V_{c}, 2}$ corresponds to the $V_{c, 2}=22 \mathrm{~mm} / \mathrm{rad} ; R_{V_{c}, 3}$ corresponds to the $V_{c, 3}=21 \mathrm{~mm} / \mathrm{rad} ; \Sigma_{r}=$ $60^{\circ} ; j_{21}=42 \mathrm{~mm} / \mathrm{rad}$.

characterized by the smallest values of relative velocity motion. The locus of these points is the axis $O_{0} z_{0}$ of $S_{0}\left(O_{0}, x_{0}, y_{0}, z_{0}\right)$. The kinematic cylinders for concrete kinematic and geometric parameters of the spatial rack drive are illustrated in Fig. 3. A computer program is developed, which is oriented to the synthesis and visualization of the basic elements of the scalar and vector field of the high kinematic joint, that realizes transformation $(R \leftrightarrow T)$.

Vector field of the relative velocity $\bar{V}_{12}$ is that part of three-dimensional space, where the function (7) is defined in each point of that space. Let (7), respectively (8), is presneted in the co-ordinate system $S_{0}\left(O_{0}, x_{0}, y_{0}, z_{0}\right)$ :

$$
\begin{aligned}
& \bar{V}_{12, s_{0}}=V_{12, x_{0}} \bar{i}_{0}+V_{12, y_{0}} \bar{j}_{0}+V_{12, z_{0}} \bar{k}_{0}, \\
& V_{12, x_{0}}=-y_{0}, \quad V_{12, y_{0}}=x_{0}, \quad V_{12, z_{0}}=j_{21} \cos \Sigma_{r},
\end{aligned}
$$

where $V_{12, x_{0}}, V_{12, y_{0}}, V_{12, z_{0}}$ are the scalar components of the vector $\bar{V}_{12}$ in co-ordinate system $S_{0} ; \bar{i}_{0}, \bar{j}_{0}, \bar{k}_{0}$ are the unit vectors of the co-ordinate axes of $S_{0}$.

It is known [9], that every vector field is characterized by its own vector lines. The vector lines of the vector function (14) represent curves. The tangential lines in every point $P\left(x_{0}, y_{0}, z_{0}\right)$ to these curves coincide with the direction of the vector $\bar{V}_{12}$ in the same point. They are defined by the following system of differential equations:

$$
\begin{aligned}
\frac{d x_{0}}{V_{12, x_{0}}} & =\frac{d y_{0}}{V_{12, y_{0}}}, & \frac{d x_{0}}{V_{12, x_{0}}} & =\frac{d z_{0}}{V_{12, z_{0}}} \text { or } \\
\frac{d x_{0}}{V_{12, x_{0}}} & =\frac{d y_{0}}{V_{12, y_{0}}}, & \frac{d y_{0}}{V_{12, y_{0}}} & =\frac{d z_{0}}{V_{12, z_{0}}} .
\end{aligned}
$$




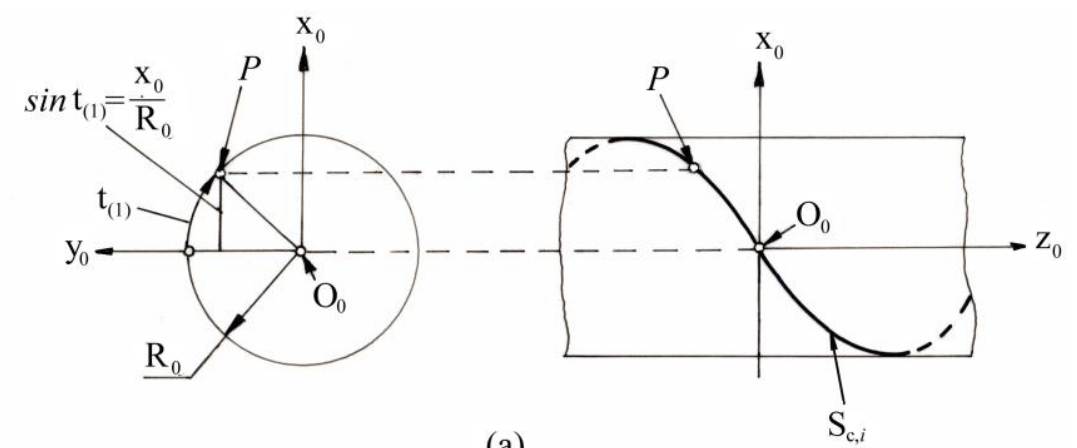

(a)

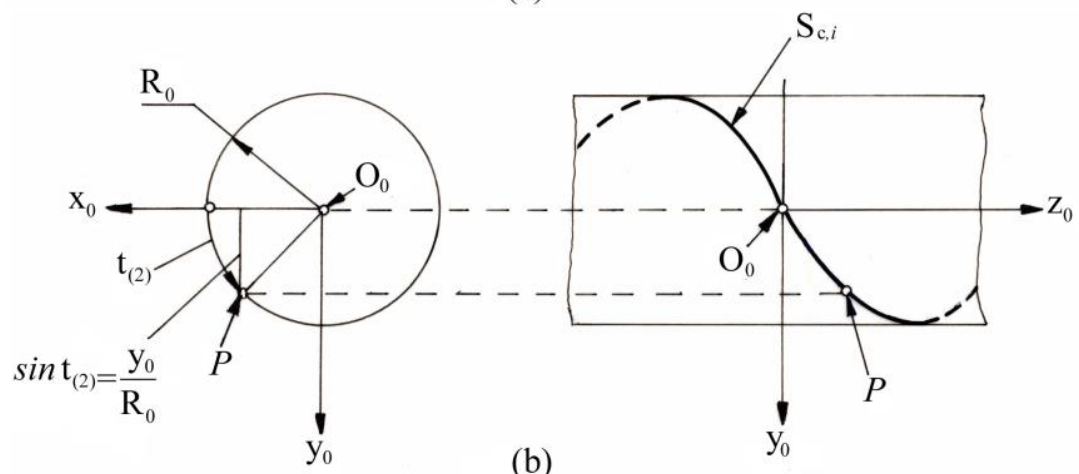

(b)

Fig. 4. Scheme of formation of the kinematic relative helices $S_{c, i}$ : a) for the angular parameter $t_{(1)}=\arcsin \left(x_{0} / R_{0}\right)$; b) for the angular parameter $t_{(2)}=\arcsin \left(y_{0} / R_{0}\right)$.

From the first system of (15) it is obtained respectively (see Fig. 4a):

$$
\begin{aligned}
& x_{0}^{2}+y_{0}^{2}=R_{0}^{2}=\mathrm{constant}, \\
& z_{0}+p_{z_{0}} t_{(1)}=C_{0_{(1)}}=\mathrm{constant},
\end{aligned}
$$

where $p_{z_{0}}=j_{21} \cos \Sigma_{r}, t_{(1)}=\arcsin \frac{x_{0}}{R_{0}}$. Analogically from the second equations set of (15) it is obtained (see Fig. 4b)

$$
\begin{aligned}
& x_{0}^{2}+y_{0}^{2}=R_{0}^{2}=\mathrm{constant}, \\
& z_{0}-p_{z_{0}} t_{(2)}=C_{0_{(2)}}=\mathrm{constant},
\end{aligned}
$$

where $p_{z_{0}}=j_{21} \cos \Sigma_{r}, t_{(2)}=\arcsin \frac{y_{0}}{R_{0}}$.

Analyzing equations systems (16) and (17), it is established that vector lines of the vector field of function $\bar{V}_{12}=\bar{V}_{12}\left(x_{0}, y_{0}, z_{0}\right)$ are family of helical lines. They are 
obtained as intersections of the family of coaxial cylinders with radius $R_{0}$ with a family of helical conoids (orthogonal Archimedean helicoids) with a helical parameter $p_{z_{0}}$. The following is fulfilled for $p_{z_{0}}$ :

$$
p_{z_{0}}=\frac{V_{12, z_{0}}}{\omega_{12}}=\frac{V_{12, z_{0}}}{\omega_{1}}=V_{12, z_{0}},
$$

because of the fact, that for the studied motions transformation $\bar{\omega}_{2}=\overline{0}$, and $\omega_{1}=$ $1 \mathrm{rad} / \mathrm{s}$. These conoids are called kinematic helical conoids, and the helical lines, described by systems (16) and (17), are called kinematic relative helices [8]. They are characterized with parameters $R_{0}, p_{z_{0}}, C_{0_{(i)}}(i=1,2)$. It is obvious, when the kinematic relative helices of the vector field $\bar{V}_{12}=\bar{V}_{12}\left(x_{0}, y_{0}, z_{0}\right)$ are defined, then a relative helical motion of the conjugated surfaces is defined, which arises in the process of motions transformation $(R \leftrightarrow T)$. It can be shown easily, that if $R_{0}=0$ is substituted in the equations systems (16) and (17), they describe the equation of the axis $O_{0} z_{0}$ in the fixed co-ordinate system $S_{0}$.

Let the second equation from (16) and (17) is presented respectively in the form

$$
\begin{aligned}
& z_{0}=C_{0_{(1)}}-p_{z_{0}}\left[k \pi+(-1)^{k} t_{(1)}\right], \\
& k \in Z, \quad t_{(1)} \in(0,2 \pi)
\end{aligned}
$$

and

$$
\begin{aligned}
& z_{0}=C_{0_{(2)}}+p_{z_{0}}\left[k \pi+(-1)^{k} t_{(2)}\right], \\
& k \in Z, \quad t_{(2)} \in(0,2 \pi),
\end{aligned}
$$

where $Z$ is the set of integers of the whole numbers and their additive inverses. From the first equations of (16) and (17), if $R_{0}=0$ then $x_{0}=y_{0}=0$. The second equations, written in the form (19) and (20) are parametric equations of the axis $O_{0} z_{0}$, which is also an axis of family of kinematic relative helices.

Considering the values of the scalar components of the sliding velocity vector $\bar{V}_{12}$ in $S_{0}\left(O_{0}, x_{0}, y_{0}, z_{0}\right)$, from (14) for the value of sliding velocity it can be written

$$
V_{12, s_{0}}=\sqrt{x_{0}^{2}+y_{0}^{2}+\left(j_{21} \cos \Sigma_{r}\right)^{2}} .
$$

From (21) it is obvious, that the sliding velocity vector $\bar{V}_{12}$ at an arbitrary point from the axis $O_{0} z_{0}$ has the minimum value, i.e.

$$
V_{12, \min }=V_{12, z_{0}}=p_{z_{0}}=j_{21} \cos \Sigma_{r}
$$




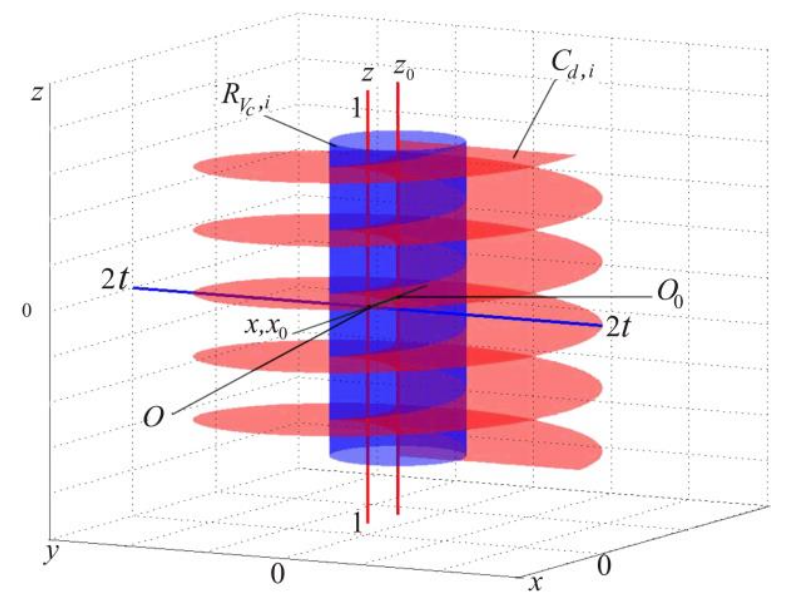

Fig. 5. Kinematic scheme of the relative helices generation of the vector function $\bar{V}_{12}=$ $\bar{V}_{12}(x, y, z)$ : kinematic cylinder $R_{V_{c}, i}$ and kinematic helical conoid $C_{d, i}$, when $j_{21}=$ $42 \mathrm{~mm} / \mathrm{rad} ; V_{c}=44 \mathrm{~mm} / \mathrm{rad} ; \Sigma_{r}=60^{\circ}$.

For this reason the axis $O_{0} z_{0}$ is called a minimum kinematic relative helix. According to (18) and (22), if $\Sigma_{r} \in(-\pi / 2, \pi / 2)$, then $p_{z_{0}}>0$, and therefore equations (16) describe all the right-oriented kinematic relative helices, and when $\Sigma_{r} \in$ $(\pi / 2,3 \pi / 2)$, then $p_{z_{0}}<0$, so (16) and (17) define all the left-oriented kinematic relative helices.

For $\Sigma_{r}=-\pi / 2$ and $\Sigma_{r}=\pi / 2 \Rightarrow p_{z_{0}}=0$ and hence, from a kinematic view point, spatial motions transformation of type $(R \leftrightarrow T)$ is transformed into motion in a plane. In this case the kinematic relative helices are transformed into relative kinematic circles.

The kinematic surfaces of level and kinematic relative helical lines of the rack transmission with concrete kinematic and geometric parameters are illustrated in Fig. 5.

\subsection{NORMALIZED KINEMATIC RELATIVE HELICES}

The value of sliding velocity vector is a characteristic that takes part in the development of important quality criteria, which are applied to the synthesis of mechanical motions transformers, by using high kinematic joints $[1,8]$. This requires normalizing the module of the sliding velocity vector. For this reason, in this study an approach is applied, based on the symbiosis between the vector and scalar field of the function $\bar{V}_{12}=\bar{V}_{12}\left(x_{0}, y_{0}, z_{0}\right)$. It is realized when in (16) (in (17) respectively) is substituted

$$
R_{0}=\sqrt{V_{c}^{2}-j_{21}^{2} \cos ^{2} \Sigma_{r}}=\sqrt{V_{c}^{2}-p_{z_{0}}^{2}} .
$$


Then, the normalized kinematic relative helices are described by the following sets of equations:

$$
\begin{aligned}
& x_{0}^{2}+y_{0}^{2}=\left(V_{c}^{2}-p_{z_{0}}^{2}\right), \\
& z_{0}+p_{z_{0}} t_{0_{(1)}}=C_{0_{(1)}}, \\
& p_{z_{0}}=j_{21} \cos \Sigma_{r}, \quad t_{0_{(1)}}=\arcsin \frac{x_{0}}{R_{0}}
\end{aligned}
$$

and

$$
\begin{aligned}
& x_{0}^{2}+y_{0}^{2}=\left(V_{c}^{2}-p_{z_{0}}^{2}\right), \\
& z_{0}-p_{z_{0}} t_{0_{(2)}}=C_{0_{(2)}}, \\
& p_{z_{0}}=j_{21} \cos \Sigma_{r}, \quad t_{0_{(2)}}=\arcsin \frac{y_{0}}{R_{0}} .
\end{aligned}
$$

\section{KinEMATIC PITCH CONFIGURATIONS}

\subsection{ESSENCE AND CONTENT OF "PITCH CONFIGURATIONS"}

The elements of the kinematic theory of spatial transformation $(R \leftrightarrow T)$, examined in this study are applied for defining the geometric and kinematic nature of the main constructive characteristics (primitives) of the mathematical models, used for the synthesis of spatial rack drives. They are known under the name "pitch configurations" [8] and they treat an actual direction in the theory of gearing.

From a relatively long time, in the theory of spatial gearing are used together the terms "primary surfaces" $[3,10]$ and term "pitch surfaces/operating pitch surfaces" $[4,5,11]$. To a greater extent in the cited literature with these two terms - primary and pitch surfaces are defined similar concepts.

In the late 20th century Prof. F. Litvin, a professor of mechanical engineering at the University of Illinois at Chicago - USA and an active researcher and consultant in the field of gears, remained almost unchange the shown by him concept of the primary surfaces in [3]. In [5], primary surfaces are called "operating pitch surfaces", in relation to their practical application in the design of spatial gear sets with crossed axes, unlike the axodes of movable link:

"Operating pitch surfaces represent: (i) two cylinder for worm-gear drive and helical gears with crossed axes, and (ii) two cones for a hypoid gear drives. The chosen surfaces, that are called in the technical literature "operating pitch surfaces" must satisfy the following requirements:

(i) the axes of cylinders (cones) have to form the same twist angle and to be at the same shortest distance, as for the gears to be designed. 
(ii) the cylinders (cones) must be in tangency at the mean point of contact of the surfaces of the gears to be designed.

(iii) the relative sliding velocity $\bar{V}^{(12)}$ at point $P$ of tangency of the cylinders (cones) must lie in the plane, that is tangent to the cylinders (cones) and $\bar{V}^{(12)}$ must be directed along the common tangent to the helices of the gears to be designed. The term "helix" is a conventional one. Actually, we have to consider a spatial curve that belongs to the operating cylinders (cones) and represents the line of intersection of the gear tooth surface with the operating cylinders (cones). For the case of helical gear, a cylindrical worm, this line of intersection is indeed a helix. For the case of spiral bevel gears and hypoid gears, the line of intersection is a spatial curve that differs from a helix and might be represented with complicated equations.

(iv) Point $P$ of tangency of operating pitch cylinders (cones) will be simultaneously the point of tangency of gear tooth surfaces if the surfaces possess a common normal $\bar{N}$ at $P$ and $\bar{N}$ is perpendicular to $\bar{V}^{(12)} \ldots .$.

Cited above present version of the idea for the pitch surfaces is oriented exclusively to the practice of the worm, helical and hypoid gears synthesis and design. A similar meaning have the conditions for the existence of the "primary surfaces", defined in [10].

The studies, oriented for refining of the content and the name of this concept are summarized in [8]. There the existence of kinematic and geometric pitch circles and pitch surfaces forming the common name pitch configurations (kinematic and geometric) is justified.

The brief overview of the researches in this field shows the crucial importance of the characteristics of kinematic geometry of the processes of spatial motions transformation, as basic primitives of mathematical models for the synthesis of adequate transmissions.

\subsection{RACK DRIVES KINEMATIC PITCH CONFIGURATIONS}

Kinematic pitch surfaces, are surfaces which are obtained as envelopes of the normalized relative helices of the vector function $\bar{V}_{12}=\bar{V}_{12}\left(x_{0}, y_{0}, z_{0}\right)$ for their relative motion towards the axes of the transformation of the type $(R \leftrightarrow T)$ [7]. As it was already clarified, the sets of normalized relative helices are placed on concretely defined kinematical cylinders of level. In other words, the kinematic pitch surfaces (for the studied case) should be treated as envelopes of the corresponding kinematic cylinders of level. These surfaces are firmly connected with the co-ordinate systems $S_{i}\left(O_{i}, x_{i}, y_{i}, z_{i}\right)(i=1,2)$. 
In relation to the mentioned above, the system of equations (11) is presented as

$$
\begin{aligned}
& x_{0}=R_{0} \sin t, \quad y_{0}=R_{0} \cos t, \\
& z_{0}=H, \quad H \in(-\infty,+\infty), \quad t=\arcsin \frac{R_{0}}{x_{0}} .
\end{aligned}
$$

Let the canonical form (22) of kinematical cylinders of level is written in the coordinate systems $S_{i}\left(O_{i}, x_{i}, y_{i}, z_{i}\right)(i=1,2)$ (taking into considerations the symbols in Fig. 2). For this purpose, the following matrix equalities are used:

$$
\left\|x_{1} \quad y_{1} \quad z_{1} \quad t_{1}\right\|^{T}=M_{S_{1} S_{0}}\left\|x_{0} \quad y_{0} \quad z_{0} \quad t_{0}\right\|^{T},
$$

where:

$$
M_{S_{1} S_{0}}=\left\|\begin{array}{llll}
\cos \varphi_{1} & \sin \varphi_{1} & 0 & -j_{21} \sin \Sigma_{r} \cos \varphi_{1} \\
-\sin \varphi_{1} & \cos \varphi_{1} & 0 & +j_{21} \sin \Sigma_{r} \sin \varphi_{1} \\
0 & 0 & 1 & 0 \\
0 & 0 & 0 & 1
\end{array}\right\|
$$

and

$$
\left\|x_{2} \quad y_{2} \quad z_{2} \quad t_{2} \quad\right\|^{T}=M_{S_{2} S_{0}}\left\|x_{0} \quad y_{0} \quad z_{0} \quad t_{0}\right\|^{T}
$$

where

$$
M_{S_{2} S_{0}}=\left\|\begin{array}{cccc}
1 & 0 & 0 & -j_{21} \sin \Sigma_{r} \\
0 & 1 & 0 & j_{21} \sin \Sigma_{r} \varphi_{1} \\
0 & 0 & 1 & j_{21} \cos \Sigma_{r} \varphi_{1} \\
0 & 0 & 0 & 1
\end{array}\right\|
$$

When (27) and (28) are solved, it is obtained respectively

$$
\begin{aligned}
& x_{1}=R_{0} \sin \left(t+\varphi_{1}\right)-j_{21} \sin \Sigma_{r} \cos \varphi_{1}, \\
& y_{1}=R_{0} \cos \left(t+\varphi_{1}\right)+j_{21} \sin \Sigma_{r} \sin \varphi_{1}, \\
& z_{1}=H, \quad H \in(-\infty,+\infty)
\end{aligned}
$$

and

$$
\begin{aligned}
& x_{2}=R_{0} \sin t-j_{21} \sin \Sigma_{r}, \\
& y_{2}=R_{0} \cos t+j_{21} \sin \Sigma_{r} \varphi_{1}, \\
& z_{2}=H+j_{21} \cos \Sigma_{r} \varphi_{1}, \quad H \in(-\infty,+\infty) .
\end{aligned}
$$

The equation systems (29) and (30) describe families of enveloped kinematic surfaces of level. Their envelopes, treated as a locus of the contact lines of enveloped and 


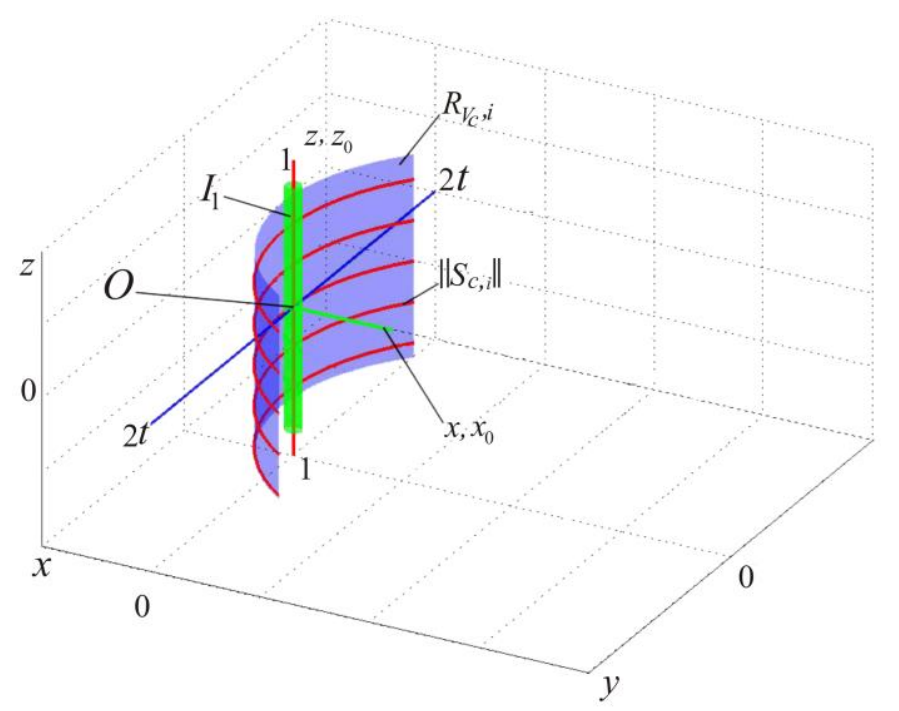

Fig. 6. Kinematic pitch cylinder $I_{1}$ with radius $\left|R_{0}-j_{21} \sin \Sigma_{r}\right|: R_{0}=25 \mathrm{~mm} / \mathrm{rad} ; j_{21}=$ $42 \mathrm{~mm} / \mathrm{rad} ; \Sigma_{r}=60^{\circ} ; R_{V_{c}, i}-$ kinematic cylinder of level; $S_{c, i}$ - normalized relative helix.

enveloping surfaces, which are written in the co-ordinate systems $S_{i}\left(O_{i}, x_{i}, y_{i}, z_{i}\right)$ $(i=1,2)$, are obtained, when the following condition is added to (29) and (30):

$$
\bar{e}_{0, s_{i}} \bar{V}_{i, s_{i}}=0, \quad(i=1,2),
$$

where $\bar{e}_{0, S_{i}}$ is the unit vector to an arbitrary kinematic cylinder of level in its arbitrary point $P_{0}\left(x_{0}, y_{0}, z_{0}\right) ; \bar{V}_{i}-$ transference velocity vectors $(i=1-$ rotational and $i=2$ - translational) of an arbitrary point $P_{0}$, of an arbitrary kinematic cylinder of level; $S_{i}$ ( $i=1,2)$ - co-oridnate systems, connected with movable links $i=1$, realizing rotation motion with angular velocity $\bar{\omega}_{1}$, and $i=2$, realizing translation with velocity $\bar{V}_{2}$. Then, the searched envelopes have the following analytical expression:

$$
\begin{aligned}
& x_{1}^{2}+y_{1}^{2}=\left(R_{0} \mp j_{21} \sin \Sigma_{r}\right)^{2} \\
& z_{1}=H, \quad H \in(-\infty,+\infty)
\end{aligned}
$$

and

$$
\begin{aligned}
& x_{2}= \pm R_{0}-j_{21} \sin \Sigma_{r}, \\
& \cot \Sigma_{r} y_{2}-z_{2}+H=0, \quad H \in(-\infty,+\infty) .
\end{aligned}
$$

Hence, kinematic pitch surfaces, for the rack drives, are two pairs of the rotational pitch cylinders (32) and contacting with them corresponding pitch planes (33): 


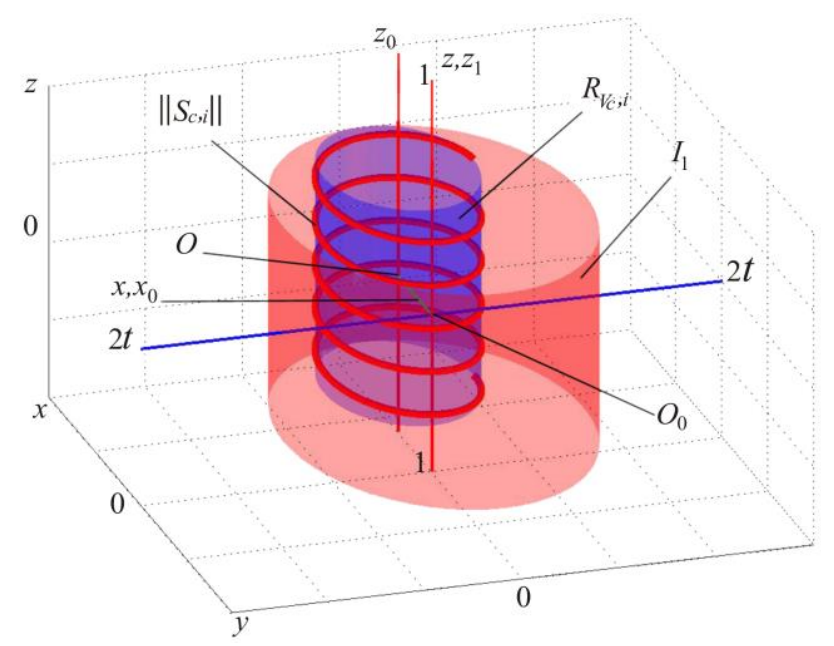

Fig. 7. Kinematic pitch cylinder $I_{1}$ with radius $\left|R_{0}+j_{21} \sin \Sigma_{r}\right|: R_{0}=38 \mathrm{~mm} / \mathrm{rad} ; j_{21}=$ $42 \mathrm{~mm} / \mathrm{rad} ; \Sigma_{r}=60^{\circ} ; R_{V_{c}, i}$ - kinematic pitch cylinder of level; $S_{c, i}-$ normalized relative helix.

$$
\begin{aligned}
& x_{1}^{2}+y_{1}^{2}=\left(R_{0}-j_{21} \sin \Sigma_{r}\right)^{2}, \quad z_{1}=H, \\
& x_{2}=R_{0}-j_{21} \sin \Sigma_{r}, \\
& \cot \Sigma_{r} y_{2}-z_{2}+H=0, \quad H \in(-\infty,+\infty), \\
& x_{1}^{2}+y_{1}^{2}=\left(R_{0}+j_{21} \sin \Sigma_{r}\right)^{2}, \quad z_{1}=H, \\
& x_{2}=-\left(R_{0}+j_{21} \sin \Sigma_{r}\right), \\
& \cot \Sigma_{r} y_{2}-z_{2}+H=0, \quad H \in(-\infty,+\infty) .
\end{aligned}
$$

It is evident from (30) and (31), that when $R_{0}=0$ the axodes of the rack mechanism are obtained. These axodes are cylinder and a plane, which are tangent to the zero kinematic cylinder $O_{0} z_{0}$. The kinematic pitch cylinders are illustrated by using the developed computer program. They are defined for the concrete case of motions, transformation by the first two equations from the systems (34) (see Fig. 6) and (35) (see Fig. 7).

In Fig. 8, the axodes (cylinder and plane) of spatial rack drive are visualized.

From the realized and illustrated study, it is obvious the following:

- each common point of axodes (cylinder and plane) and pitch surfaces (cylinder and plane) coincides with a point from the normalized kinematic relative helix;

- the axis of the relative helices $O_{0} z_{0}$, that coincides with the zero kinematic relative helix, is parallel to the geometric axis $1-1$ of the rotating link $i=1$; 


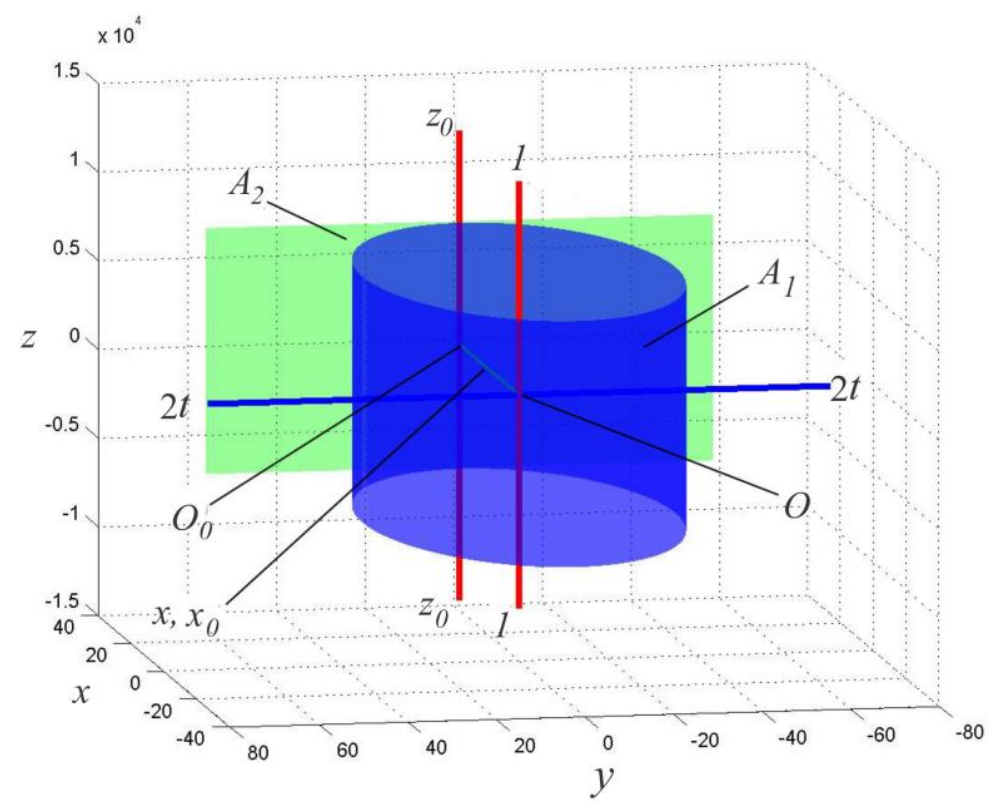

Fig. 8. Axodes of spatial rack drive (cylinder $A_{1}$ and plane $\left.A_{2}\right)-j_{21}=42 \mathrm{~mm} / \mathrm{rad} ; \Sigma_{r}=$ $60^{\circ}$.

- the angle of inclination of each representative of the family normalized relative helices, at each common point of the pitch surfaces (including for axodes), is a constant one.

The defined special features, relative to the kinematic pitch surfaces, allow to be determined the kinematic pitch circle and pitch line. They are the cross sections of the kinematic pitch cylinder, which geometric axis is parallel to the axis of rotation and line, lying in the kinematic pitch plane, parallel to the directrix of translation. The circle and the plane have a common point, which is a common point of the normalized relative helix.

The corresponding pairs, kinematic pitch cylinder and kinematic pitch plane and the pair kinematic pitch circle and kinematic pitch line form the kinematic pitch configurations.

\section{COMPUTER PROGRAM FOR SYNTHESIS AND VISUALIZATION OF BASIC KINEMATIC CHARACTERISTICS OF SPATIAL MOTIONS TRANSFORMATION $(R \leftrightarrow T)$}

The program, described below, is oriented towards the study and visualization of the kinematic character of the motions transformation of type "rotation into transla- 
tion". At the same time, the contained in the program modules are able to ensure the synthesis of kinematic pitch configurations. These configurations can be treated as geometric-kinematic primitives, that are basic for the design of the spatial rack drives. They are analytically defined and the approach for their visualization will be illustrated here.

The computer program is based on the MATLAB software [12]. It contains a description and declaration of the input parameters and functions. When they are executed, the different kinematic characteristics and pitch configurations of the studied mechanisms are obtained.

The input parameters of the program are:

- Geometric input parameters:

- crossed angle of the axes of motions transformation $1-1$ and $2 t-2 t-$ $\delta_{r} \in(0, \pi)[\mathrm{rad}]$

- angular parameter - $t[\mathrm{rad}]$;

- Kinematic input parameters:

- velocity ratio - $j_{12}[\mathrm{rad} / \mathrm{mm}]$ or $j_{21}[\mathrm{~mm} / \mathrm{rad}]$;

- module of the given sliding velocity $-V_{12}[\mathrm{~mm} / \mathrm{s}]$;

- module of the angular velocity $-\omega_{1}[\mathrm{rad} / \mathrm{s}]$.

Instead the last two parameters, it can be given $V_{c}=\omega_{1}^{-1} V_{12}[\mathrm{~mm} / \mathrm{rad}]$.

Structurally, the computer program consists of one main and four executive subprograms. The execution of each sub-program is realized by importing in the main program a key $K$, which can take values as it follows:

- $K=1$. The result of execution of the program here is drawing of the rotation axis $1-1$, of the link $i=1$ and axis (direction) of translation $2 t-2 t$ of the link $i=2$ of spatial rack mechanisms, kinematic cylinders of level $R_{V_{c}, i}$, including zero kinematic cylinder $z_{0}$. When the input geometric and kinematic parameters are suitably chosen, there are obtained variously positioned in the space families of kinematic surfaces of level (see Fig. 3).

- $K=2$. In this case, the graphic image contains the axes $1-1$ and $2 t-2 t$, kinematic cylinders of level $R_{V_{c}, i}$, kinematic conoids $C_{d, i}$, including axis $z_{0}$ of the relative helical motion (zero cylinder of level) (see Fig. 5).

- $K=3$. In this case, the result of execution of the sub-program is a graphic image, that contains axes $1-1,2 t-2 t$ and axis of relative helical motion 
$z_{0}$, around which is defined a concrete normalized relative helix $\left\|S_{c, i}\right\|$ and the envelope of the family of normalized relative helices, which are the kinematic pitch cylinders (see Figs. 6 and 7).

\section{CONCLUSION}

It is presented the structure, geometric and kinematic similarity of the processes of spatial motions transformation of type $(R \leftrightarrow R)$ and $(R \leftrightarrow T)$. On the basis of the vector analysis of the sliding velocity between the elements of high kinematic joints, that realize transformation $(R \leftrightarrow T)$, the analytical type of kinematic cylinders of level and kinematic relative helices is obtained. The basic kinematic characteristics - kinematic pitch configurations and axodes that characterize the mechanics of a spatial motions transformation by means of three-link mechanical systems, with high kinematic joints, are defined analytical in this study. They have an important essence for the development of the mathematical models, applicable to the synthesis of these class motions transformers.

A computer program, oriented to the analysis, synthesis and visualization of the basic kinematic characteristics of the studied type motions transformation, is developed.

\section{REFERENCES}

[1] Abadjieva, E. Mathematical Models of the Kinematic Processes in Spatial Rack Mechanisms and their Application, Ph. D Thesis, Bulgaria, Sofia, Institute of Mechanics- BAS, 2010, 165.

[2] Abadjieva, E. Spatial Rack Drives, Mathematical Modelling for Synthesis, VDM Verlag Dr. Müller e.K., 2011, 72.

[3] Litvin, F. Theory of Gearing, Moskow, Nauka, 1968, 584 (in Russian).

[4] Litvin, F. Theory of Gearing, NASA Reference Publication 1212, AVSCOM Technical Report 88-C-035, US, Washington, Government Printing Office, 1989, 470.

[5] Litvin, F. Gearing Geometry and Applied Theory, PTR Prentice Hall, A Paramount Communication Company, Englewood Eliffs, New Jarsy 07632, 1994, 724.

[6] Abadjiev, V., E. Abadjieva. Geometry Kinematical Correspondence of Spatial Transformations "Rotation to Rotation" and "Rotation to Translation", Int. Conf. on Manufacturing Systems, 7-8 October 2004, Romania, Bucharest, 2004, 147-150.

[7] Abadjiev, V., E. Abadjieva. Vector Analysis of the Sliding Velocity Function of Rack Drives. J. of Theoretical and Applied Mechanics, Sofia, 37 (2007), No. 4, 15-24.

[8] Abadjiev, V. Gearing Theory and Technical Applications of Hyperboloid Mechanisms, Sc. D. Thesis, Sofia, Bulgarian Academy of Sciences, Institute of Mechanics, 2007, 309 (In Bulgarian).

[9] Korn, G., T. Korn. Mathematical Handbook for Scientists and Engineers. Definitions, Theorems, Formulas, Moskow, Nauka, 1973, 831. 
[10] Minkov, K. S. Mechano-Mathematical Modeling of Hyperboloid Gear Sets, Sc. D. Thesis, Sofia, Bulgarian Academy of Sciences, Institute of Mechanics, 1986, 303.

[11] Litvin, F., K. Petrov, V. Ganchin. The Effect of Geometrical Parameters of Hypoid and Spiroid Gears in Its Quality Characteristics. Transaction of ASME. Journal of Engineering for Industry, 96 (1974), No. 1, Series B, 330-334.

[12] Matlab, MathWorks, Release2006B. 